CASE REPORT

\title{
DYKE-DAVIDOFF-MASSON SYNDROME-A Rare Cause of Cerebral Hemiatrophy in a 17-Years-Old Ethiopian Patient: A Case Report
}

\author{
Biniyam Alemayehu Ayele ${ }^{1^{*}}$, Yared Zenebe Zewde ${ }^{1}$
}

\begin{abstract}
OPEN ACCESS
Citation: Biniyam Alemayehu Ayele, Yared Zenebe Zewde. DYKEDAVIDOFF-MASSON SYNDROME-A Rare Cause of Cerebral Hemiatrophy in a 17-Years-Old Ethiopian Patient: A Case Report. Ethiop J Health Sci. 2018;29(2):287.

doi:http://dx.doi.org/10.4314/ ejhs.v29i2.16

Received: September 21, 2018

Accepted: November 5, 2018

Published: March 1, 2019

Copyright: (C) 2019 Biniyam Alemayehu Ayele, et al. This is an open access article distributed under the terms of the Creative Commons Attribution License, which permits unrestricted use, distribution, and reproduction in any medium, provided the original author and source are credited.

Funding: Nil

Competing Interests: The authors declare that this manuscript was approved by all authors in its form and that no competing interest exists.

Affiliation and Correspondence:

${ }^{1}$ Department of Neurology, School of Medicine, College of Health Sciences, Addis Ababa University *Email: biniyam.a7@gmail.com
\end{abstract}

\section{ABSTRACT}

BACKGROUND: Dyke-Davidoff-Masson syndrome (DDMS) is a rare disease with unknown frequency, mainly presented with seizures, facial asymmetry, contralateral hemiparesis and mental retardation. Often, resulting from brain injury due to a multitude of causes, especially in early life, associated with birth asphyxia. Radiological findings include cerebral hemiatrophy/hypoplasia, calvarial thickening, and hyperpneumatization of the frontal sinuses.

CASE PRESENTATION: We report the case of a 17-year-old male patient who presented to Neurology Clinic with complaints of left side body weakness, walking difficulty and poorly controlled seizure for the past 6 years. Brain MRI revealed atrophy of the right cerebral hemisphere.

CONCLUSION: Dyke-Davidoff-Masson syndrome should be suspected in any patients who present with classical features and brain imaging showing hemiatrophy. Early identification and treatment is important in such patients, as it can improve patients prognosis and quality of life.

KEYWORDS: Dyke-Davidoff-Masson syndrome, Seizure, Cerebral hemiatrophy,Magnetic resonance imaging

\section{INTRODUCTION}

Dyke-Davidoff-Masson syndrome (DDMS) is a rare disorder which is mainly seen in the pediatrics age group (1). It is mainly attributed to brain insult during neonatal/perinatal period, resulting in hemiatrophy or hypoplasia of the affected hemisphere (1). Patients mainly present with symptom complexes like, hemiparesis, facial asymmetry, seizure disorder and intellectual disability (2). Due to its rarity, most physicians miss the diagnosis. Therefore, we report this case in an effort to improve the knowledge gaps regarding DDMS, especially in setups like Ethiopia, as this is the first case report of its kind, to the best of the authors' knowledge. 


\section{CASE PRESENTATION}

A 17-year-old right-handed male patient from rural part of Ethiopia presented to our hospital with the complaints of left side body weakness, walking difficulty and frequent convulsion for 6 years. Six years back, the symptoms started with abnormal body movement associated with loss of consciousness, drooling of saliva and up rolling of both eyes. For these symptoms, he was evaluated at local health facility and diagnosed with epilepsy and started on phenobarbitone of unspecified dose. Later, he started to develop progressive left side hemibody weakness and gait difficulty. Despite frequent anticonvulsant dose escalation at a local health center, the seizure was not controlled; so he was referred to our facility for further management. He dropped out of school because of his left hemibody weakness and difficulty to walk, but he denied cognitive problems. There was no history of significant perinatal and antenatal complications. He was born at home as a full term baby via normal vaginal delivery. He had normal childhood development. On neurologic examination, he had spastic left side hemiparesis with brisk deep tendon reflex and extensor plantar response on the same side, Otherwise normal cognition, cranial nerves and sensory system. He had mild atrophy of left hand intrinsic muscles with clenched fist; normal examination on right hemibody (Figure 1). There were no port wine skin lesions on the face or other cutaneous lesions on skin examination. All routine tests including CBC, serum electrolyte, renal and liver function test, blood sugar, syphilis and HIV test were normal. Brain MRI showed atrophy of the right cerebral white matter with thinning of the cortical gyri and ex vacuo dilatation of right lateral ventricle, with compensatory hyperpneumatization of the right side frontal sinus and normal left cerebral hemisphere (Figure 2). Electroencephalogram (EEG) showed continuous epileptogenic discharges from the right side of the brain in the form of periodic lateralized epileptiform discharge (PLED'S) (Figure 3).

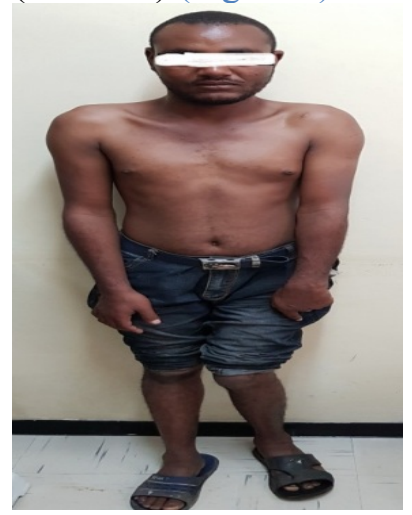

Figure 1: Left side hemiparesis, with flexed left wrist and clenched fist
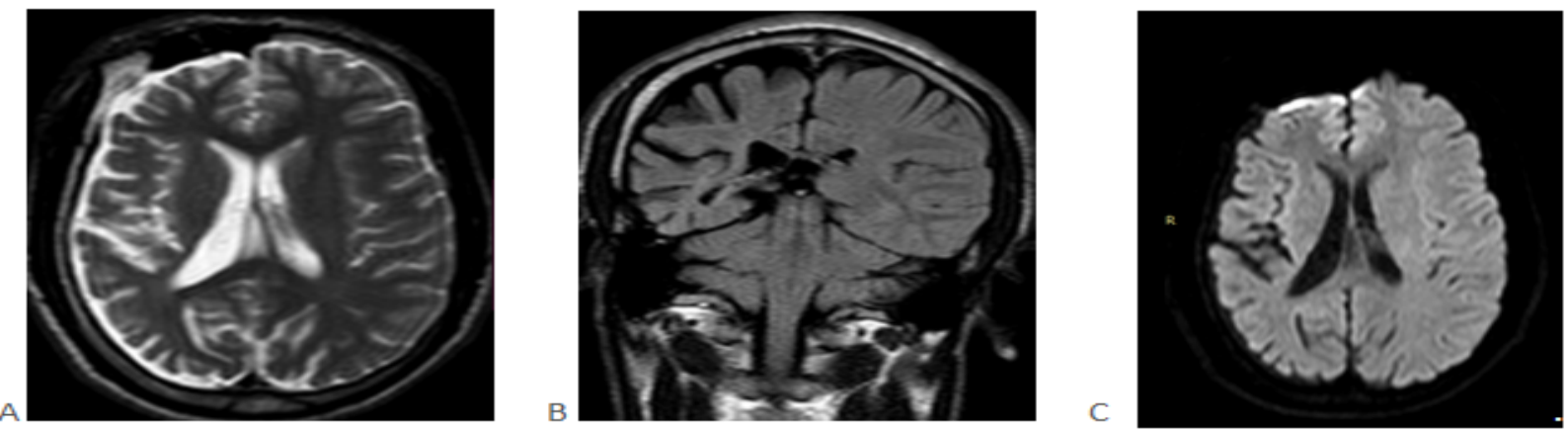

Figure 2: A) Axial T2W b) Coronal FLAIR C) Axial DWI; showed atrophy of the right cerebral white matter with thinning of the cortical gyri and ex vacuo dilatation of lateral ventricles, with hyperpneumatization of right side frontal sinus 


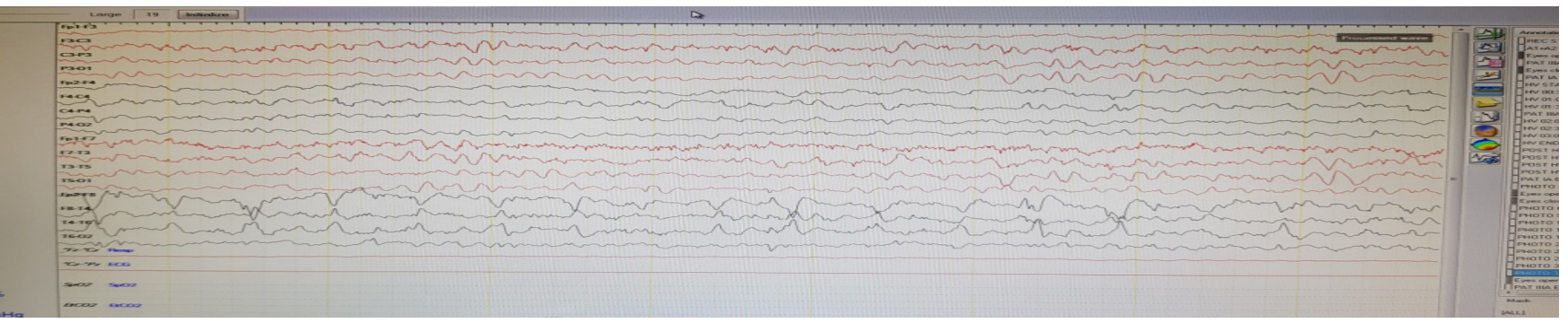

Figure 3: EEG showing PLEDS seen over right hemispheric region

Note: EEG Electroencephalogram PLEDs Period Lateralized Epileptiform Discharges

Table 1: Comparing Brain MRI of typical DDMS patient with other potential mimickers

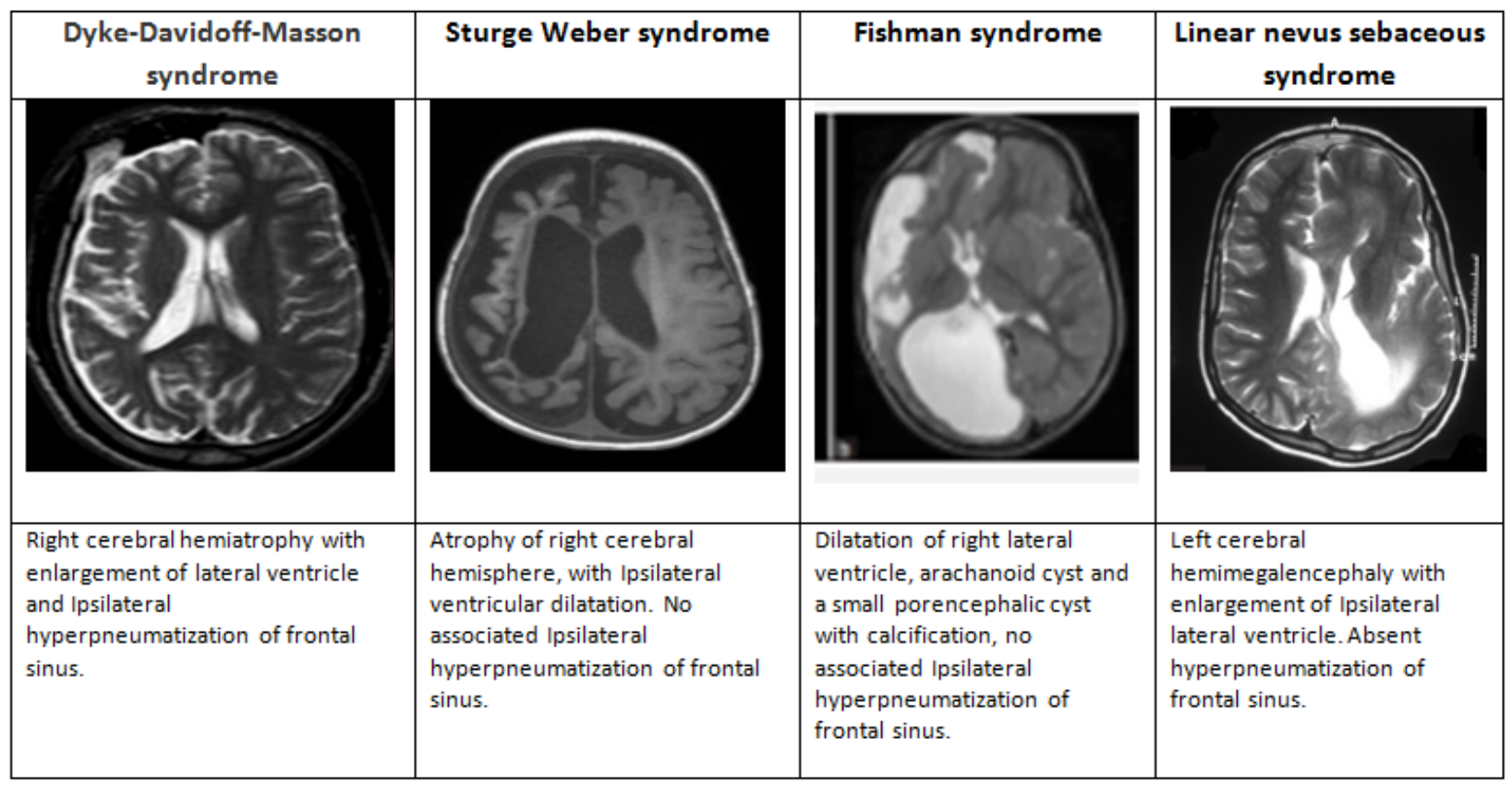

Once the diagnosis was made, our patient's seizure was controlled following optimization of his anticonvulsant medication. In addition the patient was also started on rehabilitation therapy to improve his weakness and gait abnormality.

\section{DISCUSSION}

In 1933, Dyke, Davidoff and Masson described 9 patients with clinical characteristics of hemiparesis, facial asymmetry, seizures and mental retardation noted to have pneumatoencephalographic changes on skull radiograph (3). Both sexes and any of the hemispheres may beaffected but male gender and left hemisphere involvement are more frequent. Age of presentation depends on time of neurologic insult, and characteristic changes may be seen only in adolescence. There are reports of an adult onset of DDMS (4), as our patient is now 17 -year old, even though the symptoms started when he was 11-year old, which is consistent with most of the cases reports on DDMS. Common causes include congenital anomalies, perinatalhypoxia, intracranial hemorrhage, and infectionsat early infancy (5). However our patient's perinatal history was non-revealing with normal developmental milestone and only diagnosed after brain MRI revealed the peculiar features of DDMS. 
Both CT scan and MRI are helpful in diagnosing DDMS, the hallmark imaging features for DDMS include cerebral hemiatrophy/hypoplasia, hyperpneumatization of the Para nasal sinuses and compensatory osseous hypertrophy. These features will become more evident with time, as the patient gets older; like in our patient where there are radiological evidences of brain injury occurring during the intrauterine period or before the age of 3. In addition, our patient's EEG showed periodic lateralized epileptiform discharge (PLED'S); which is a non-specific EEG abnormality, suggestive of focal cerebral lesion..

DDMS has many differential diagnoses, including, Sturge Weber syndrome (SWS), which is one of neurocutaneous syndromes. It present with port-wine staining on the face, seizure, glaucoma and leptomeningial angiomatosis on brain MRI. Therefore, since our patient had none of these symptoms, SWS was less likely. Fishman syndrome is a rare congenital neurocutaneous disease that commonly involves ectomesodermal tissues such as the eye, skin and central nervous system. It often associated with intracranial lipoma on brain MRI. Since our patients has no ocular or skin manifestations and no evidence of lipoma was seen on brain MRI, this is less likely diagnosis. Linear nevus sebaceous syndrome (LNSS) is a rare, sporadic neurocutaneous syndrome characterized by a linear sebaceous nevus of Jadassohn, mental retardation, and, seizures. In addition, patients may have hemiparesis. Since our patient has no cutaneous features and no cognitive problem LNSS is unlikely diagnosis of our patient.

Prognosis of DDMS is better if hemipares occurs after the age of 2 years and absence of prolonged or recurrent seizures. Children with intractable disabling seizures and hemiplegia are the potential candidates for hemispherectomy with a success rate of $85 \%$ in carefully selected cases.
In conclusion, to the best of our knowledge, this is the first adult case of DDMS reported from Ethiopia. It is important to consider DDMS, as a possible differential diagnosis, in patients presented with hemiparesis, facial asymmetry and seizure disorder with brain imaging suggestive of hemispheric atrophy/hypoplasia.

\section{ACKNOWLEDGMENT}

We would like to acknowledge the Department of Neurology.

\section{ETHICS APPROVAL AND CONSENT TO PARTICIPATE}

The authors' institution does not require ethical approval for publication of a single case report. Written informed consent was obtained from the patient. Written informed consent for publication of clinical details and images was obtained from the patients.

\section{REFERENCES}

1. Verma R, Sahu R. Dyke-Davidoff-Masson syndrome. BMJ Case Reports 2012;10.1136/bcr-2012-006729.

2. Roy U, Panwar A, Mukherjee A, Biswas D. Adult presentation of dyke-Davidoff-Masson syndrome: a case report. Case Rep Neurol. 2016;8:20-6.

3. Abdul Rashid AM, Md Noh MSF. DykeDavidoff-Masson syndrome: a case report. BMC Neurology, 2018; 18:76.

4. Shetty DS, Lakhkar BN, John JR. DykeDavidoff-Masson Syndrome. Neurolindia. 2003;51:136.

5. Parker CE, Harris N, Mavalwala J. Dyke-Davidoff-Masson syndrome. Five case studies and deductions from dermatoglyphics. Clin Pediatr (Phila), 1972;11:288-92. 